\title{
Preface: Saltwater and freshwater interactions in coastal aquifers
}

\author{
Vincent Post • Elena Abarca
}

Keywords Coastal aquifers · Salt-water/fresh-water relations · Salinization - Groundwater density/viscosity · Water-resources conservation

The study of coastal aquifers, i.e., aquifers that have a hydraulic connection with a saline surface-water body, forms a somewhat separate discipline in groundwater science. This type of aquifer is typically characterized by variations of groundwater salinity in space and time, which warrants special treatment of groundwater flow, groundwater chemistry and water resource management problems. The interest in the study of this type of aquifer arose both from its strategic importance for the water supply of highly populated coastal areas and its susceptibility to salinization problems. Saltwater intrusion, i.e., the displacement of fresh groundwater by groundwater with a higher salinity, has become an accepted scientific term in the research field.

Coastal aquifers and the interactions between fresh and saline groundwaters are the topic of this theme issue of Hydrogeology Journal. We decided to give this issue a twofold objective. On one hand, it is meant to present a series of papers that together demonstrate the state of the art of the research in coastal hydrogeology. On the other hand, the aim is to present a review of the extent of saltwater-intrusion problems around the world.

Received: 9 November 2009 / Accepted: 17 November 2009 Published online: 1 December 2009

(C) Springer-Verlag 2009

\author{
V. Post (区) \\ Department of Hydrology and Geo-Environmental Sciences, \\ Faculty of Earth and Life Sciences, \\ VU University, \\ De Boelelaan 1085, 1081, Amsterdam, HV, The Netherlands \\ e-mail: Vincent.post@falw.vu.nl \\ Tel.: +31-20-5987402 \\ E. Abarca \\ Department of Civil and Environmental Engineering Parsons Lab., \\ Massachusetts Institute of Technology, \\ 77 Massachusetts Avenue, Cambridge, MA 02139-4307, USA \\ e-mail: eabarca@mit.edu
}

The study of groundwater flow in coastal areas can not be considered separately from the transport of dissolved salts. This is because spatiotemporal changes in salinity give rise to density differences that control flow patterns. Consequently, equations of flow and solute transport must be solved simultaneously. The development of models that simulate flow and solute transport under variable-density conditions has been an enormous achievement. Although, by now, many sophisticated codes have become generally available, research in this field is still ongoing, which is reflected by a number of contributions to this theme issue. Voss et al. present a methodology to benchmark threedimensional (3D) variable-density flow and transport simulators. A benchmark is a test to confirm that the simulation results are correct and that the governing equations of flow and transport are correctly solved. Previously, unambiguous 3D benchmarks for unstable systems (i.e., a system in which, in some part or everywhere, the density decreases with depth) were lacking. The benchmark is based on analytically derived stability criteria for steady-state convection in a 3D box in an unstable system. Different modes of convection develop depending on the inclination of the box and the Rayleigh number. By comparing the results of the variable-density code with the analytically derived stability criteria, it can be ascertained that the code correctly simulates the physics of the flow system.

An application of a model of coupled density-dependent groundwater flow, and solute and heat transport of an unstable system is presented by Hughes et al. They present a first assessment of the aquifer salinization process occurring beneath an engineered, hypersaline cooling-canal system. Their simulations show the development of salt fingers which can reach the bottom of the aquifer in times that range between a few days and 5 years, depending on the hydraulic conductivity configuration. They conclude that the temperature gradient, in this case, has a mitigating effect on the rate of aquifer salinization.

Downwards unstable salinization is also studied by Post and Simmons, who show the results of a combined laboratory tank experiment and numerical modeling study of free convective flow at the scale of individual lowpermeability structures. Their results provide insight into the flow patterns that evolve in both the aquifer material surrounding the lens and within the lens itself. In the system studied, upward vertical-flow components are 
generated within the lens, which retard salinization of the lens from above and ultimately cause salinization of the low-permeability structures from the bottom up. These effects occur already at permeability ratios between the low- and high-permeability regions for which geological formations are considered homogeneous aquifers, which implies that the use of an average permeability value will incorrectly predict the macroscopic rate of salinization by free convective fingering.

A specific analysis of the effect of heterogeneity on seawater intrusion was carried out by Kerrou and Renard. They present a systematic numerical study on the effect of a problem's dimensions in seawater intrusion in an idealized heterogeneous aquifer and show that the behavior of the intrusion wedge is different in $2 \mathrm{D}$ and $3 \mathrm{D}$. Therefore the results of a 2D study cannot be extrapolated to heterogeneous 3D systems. They propose an upscaling technique to use 2D heterogeneous hydraulic-conductivity fields to approximate 3D seawater-intrusion patterns.

While numerical codes have reached a level of sophistication that enable us to incorporate practically all relevant hydrological processes in hydrological models of coastal aquifers, their application is still hampered by several factors. These include, amongst others, computation time and data scarcity. Sanford and Pope discuss how these factors limit our ability to forecast the salinization of individual wells using a model of the Eastern Shore of Virginia, USA. They argue that even with unlimited computer power, uncertainty with respect to hydraulic parameters and the transient nature of the salinity distribution still prevents us from reconciling regional-scale modeling with local-scale predictions. Local grid refinement and interpolation between data points by means of (airborne) geophysics may bring some abatement to these problems, but we are a long way away from making accurate predictions at the local scale in regional flow systems.

Since coastal areas are densely populated, the freshwater contained in these aquifers is a vital economic resource. Aquifer over-exploitation has resulted in saltwater intrusion on many occasions during the past and the population, as well as economic growth, tends to increase the pressure on available reserves. Proper management, like the optimization of pumping schemes, is required to prevent contamination of freshwater resources by saline groundwater. In that direction, Pool and Carrera study the efficiency and the optimal design of negative barriers to minimize salinization in inland pumping wells. Negative barriers consist of a row of wells parallel to the coast that pump saltwater from the aquifer protecting inland wells from salinization. Their results show the fundamental need of studying this problem from a 3D perspective. Pumping as much saltwater as possible resulted in a nonoptimal solution. A critical pumping rate for the saltwater pumping wells is found to exist, above which the threedimensionality of the flow field causes lateral salinization of the inland freshwater wells. They provide empirical expressions for the critical pumping rate and for the corresponding maximum desalination at the freshwater wells.

With the pressure on existing freshwater resources rising, aquifer storage and recovery (ASR) techniques have the potential to increase the availability of fresh groundwater. In ASR systems, freshwater is stored in aquifers when supply exceeds demand and it is abstracted during periods of water shortage. Bakker presents a screening tool that can be used to assess the recovery efficiency (i.e., the ratio of the recovered volume of water divided by the injected volume of water) of ASR schemes. It is based on a numerical model of the radial velocity of the freshwater-saltwater interface, which is a function of the vertical coordinate and time. The computation times of the solution method are short so that the feasibility of ASR schemes can be evaluated quickly, before resorting to more comprehensive models for a particular scheme to quantify the effects of mixing and other complicating factors.

Another way to relieve the pressure on scarce fresh groundwater reserves is to resort to brackish or saline water as a resource. Desalination more and more proves to be an economically viable technique for the production of drinking water. In arid and semi-arid regions, desalination plants are being considered or are already operating; however, in more temperate climates, purification of brackish water by reverse osmosis (BWRO) is also considered. This is discussed by Stuyfzand and Raat who provide three examples of planned BWRO facilities in the Netherlands and discuss the aspects to be taken into account in the planning of such a facility. These include the selection of a suitable source aquifer, a disposal aquifer and chemical aspects, as mineral precipitation may clog membranes, wells and the injected aquifer.

Calibrated models of real systems are a critical step towards obtaining a reliable management tool. Carrera et al. review the particular problems associated with inverse modeling in seawater-intrusion problems and point out four critical issues. Two of these can be parameterized and modified during the inversion. They include the treatment of the initial conditions and the effect of aquifer bottom topography. The other two problems derive from data interpretation - heads are not informative without salinity (density) information and salinity data are sensitive to flow within the borehole. To address these, the authors suggest modeling the measurement process explicitly when possible.

Dausman et al. present an application of a model of a saline coastal aquifer that was used to test different scenarios of possible effluent migration. Calibration of the model was required for this purpose and the work presents different methodologies for reducing the runtimes of their highly parameterized 3D density-dependent model and the difficulties that need to be overcome in the process.

The characterization of the subsurface using geophysical techniques is constantly developing and allows us to map the salinity distribution of groundwater in ever- 
greater detail. Poulsen et al. use a downhole multielectrode geo-electrical system to investigate the shape of a freshwater lens beneath a coastal dune. By taking multiple measurements at different times, it was observed how the geometry of the lens changed in response to changes in sea level, wind direction and recharge. Such multi-electrode surveys provide better insight into the details of subsurface salinity distribution. A source of uncertainty resides in these methods, however, because salinity is derived from the electrical resistivity of the subsurface, and the relation between these two parameters is non-unique. A second source of uncertainty resides in the spatial distribution of the electrical resistivity, which is calculated from point measurements at the electrodes. In the paper by Henderson et al., the capabilities and limitations of resistivity imaging in a system dominated by tides is investigated. This study demonstrates that the resolving power of the method is negatively influenced by the thickness of the layer of saline water which overlies the electrodes, which severely limits the ability of this method to detect zones of submarine freshwater outflow at high tides. It is suggested that combined approaches, like the use of crosshole measurements, may help to improve the spatial resolution of the subsurface resistivity distribution.

Geophysical methods such as these allow us to develop and refine conceptual models of the groundwater situation near the coastline and on beaches. What is still largely unexplored scientific territory is the microbiology in these flow systems, especially near the transition zone of fresh and saline groundwater. Santoro reviews the aspects of microbial nitrogen transformations near the freshwatersaltwater interface and discusses the potential effect of saltwater intrusion on the microbial communities in the subsurface. Although studies suggest that the nitrogen transformation pathways will change in response to increased salinization, long-term effects on microbial $\mathrm{N}$ cycling may be difficult to predict from short-term lab and field experiments.

Pollutant loading and saltwater intrusion occur in coastal areas by means of benthic fluxes between groundwater and surface water. King et al. propose an analytical model to evaluate the volume of water that enters the porous medium during a tidal oscillation and the associated inflowing and outflowing fluxes under the limiting assumption of there being no freshwater hydraulic gradient. They compare their model against numerical experiments from the literature and apply it to the South Atlantic Bight (USA) study area.

With the aim of presenting a review of the extent of saltwater-intrusion problems around the world, a series of papers in this theme issue describes the state of coastal aquifers and the occurrence of saltwater intrusion on each continent. Recognizing that presenting a comprehensive and detailed overview on a global scale in a series of short papers is an unrealistic objective, we nevertheless feel that the overview gives insight into the particular problems of geographical regions across the globe. Moreover, it brings together a large list of literature references that interested readers may explore further.

Steyl and Dennis provide several examples of seawater intrusion in Africa. One notable aspect of their review is the aggravation of pressure on freshwater reserves and seawater intrusion by political unrest and war. Obstacles in the way of proper aquifer management are a lack of technical cooperation and inadequate monitoring networks. Political and social stability appears to be key factors to address the seawater intrusion and contamination problems that are facing the continent.

One particular geographical region that is prone to the adverse effects of sea-level rise is the group of small island countries in the Pacific Ocean. The freshwater lenses underlying these islands are the principle resource for water supply. White and Falkland first present an overview of the factors that control the shape of the freshwater lenses and the extent of seawater intrusion. Examples are then given of the response of the lenses to drivers such as climate, tides and recharge rates. From their discussion of the threats facing these valuable freshwater supplies, it becomes evident that these aquifer systems are among the most vulnerable in the world. The success rate of desalination projects has been poor due to high financial and energy costs and the high level of technical complexity. Other strategies, like clearing of deep-rooted vegetation and rainwater harvesting, appear to be more successful remedies.

Barlow and Reichard provide a summary of the different modes of seawater intrusion along the coast of the USA, Mexico and Canada. These include lateral, upward and downward intrusion patterns as well as contamination through vertical preferential pathways like open boreholes. They describe the variety of monitoring and management tools applied and provide a brief assessment of future strategies.

Bocanegra et al. present an overview of the existing information from 15 coastal aquifers in South America. The authors emphasize the variable degree of existing knowledge and management procedures. It appears that the strategic importance of a particular coastal aquifer determines the degree of hydrological information available.

Custodio provides an overview of the conceptual hydrological models of coastal aquifers in Europe. Following a historical perspective, he portrays some of the classical and best-documented European seawaterintrusion issues. He describes the main problems in deltaic areas, other unconsolidated rocks, carbonate massifs and small islands, which provides a wide scope of coastal aquifers in Europe.

Werner reviews the documented cases of seawater intrusion in Australia. The low recharge rates of this dry continent put many aquifers at the risk of over-exploitation. He notes that even though most Australian states reported cases of saltwater intrusion, detailed investigations have not always followed. Detailed studies have only been carried out where groundwater is perceived as a 
valuable economic commodity. A management strategy that has been implemented in many places where seawater intrusion occurred is artificial recharge. Which management approaches are best fit for the future seems uncertain given the uncertainty about the hydrological functioning of coastal aquifers and the potential impacts of climate change and sea-level rise.

Unfortunately, during the compilation of the articles for this theme issue, it proved impossible to include an overview of the state of coastal aquifers in Asia. This obviously is an important omission in this overview. Anyone interested in providing such an overview for future publication in Hydrogeology Journal should contact the Exective Editor.

The papers contained in this theme issue and the broad range of research topics they cover, demonstrate that coastal hydrogeology is an active and diversified branch of hydrological research. As in other hydrological areas, early-day research was principally initiated by the development of fresh groundwater resources for water supply. The saltwater intrusion that resulted from over-exploitation became a research topic in its own right. The foremost goal of many studies appears to be to devise optimal management strategies that maximize the use of fresh groundwater, whilst minimizing saltwater intrusion. Despite the tremendous progress that has been made over the past decades, any model forecast is still associated with great uncertainty. The reason for this is that the movement of groundwater and solutes is determined by processes acting both at the local or even pore scale, as well as by larger-scale processes such as regional top- ography-driven flow. Changes in boundary conditions of the systems we study occur at timescales of seconds (e.g., wave action on beaches) to millennia (i.e. coastline migration and sediment deposition). Our current generation of models is unable to reconcile these scales and it is questionable if these scales will ever be reconciled. Future research on coastal groundwater systems should therefore focus on minimizing uncertainty by increasing our understanding of the processes that control dissolved salt concentrations, especially at the local scale, and by developing quantitative frameworks that allow us to better incorporate and quantify uncertainty in our predictions.

Research on coastal aquifers is likely to have a stronger multi-disciplinary character in the future. Oceanographers have already turned to coastal aquifers looking for answers concerning nutrient and contaminant pathways to the ocean. With them, hydrogeologists share common research interests that provide a framework for cooperation. The same holds for microbiology, as the role of microbes in the ecosystem functioning of coastal aquifers is still unexplored terrain. Research of past and future climate change is yet another example of a scientific discipline that links with coastal hydrology. Finally, the development and application of coupled density-dependent flow and reactive transport codes is likely to increase in the near future, since interesting geochemical systems develop where waters of varying salinity mix, which calls for collaboration between hydrogeologists and geochemists. The outcome of these joint efforts may some day form the contents of a forthcoming theme issue on this exciting field of science. 